\title{
Determination of the Higgs boson properties with the ATLAS detector
}

\author{
Bertrand LAFORGE*† \\ LPNHE, UMR 7585, Université Pierre et Marie Curie - Paris 6, Université Denis Diderot-Paris \\ 7, CNRS/IN2P3, Paris, France \\ E-mail: laforgedlpnhe.in2p3.fr
}

\begin{abstract}
With the increase of proton-proton collision center of mass energy from 7-8 TeV to $13 \mathrm{TeV}$, LHC Run 2 will provide a higher sensitivity to Higgs boson physics than Run 1 . This paper summarizes what has been learnt by the ATLAS collaboration about the Higgs boson with LHC Run 1 data and also reports the first investigation of global properties of the Standard Model Higgs boson production and decay properties extracted based on a LHC Run 2 dataset using a combination of the two Higgs boson decay most sensitive channels at the LHC. This latter analysis combines a dataset of $13.3 \mathrm{fb}^{-1} H \rightarrow \gamma \gamma$ events with $14.8 \mathrm{fb}^{-1}$ of $H \rightarrow Z Z^{*} \rightarrow$ four leptons events. No deviation from the Standard Model expectations is observed.
\end{abstract}

38th International Conference on High Energy Physics 3-10 August 2016

Chicago, USA

\footnotetext{
* Speaker.

${ }^{\dagger}$ On behalf of the ATLAS collaboration
} 


\section{Introduction}

After the discovery of a new boson by both ATLAS [1] and CMS [2] collaborations in 2012, it became crucial to assess that this boson holds all the properties expected from the Higgs boson as predicted by the Standard Model (SM), i.e. a scalar boson coupling to bosons and fermions with very well predicted coupling constants once its mass is known.

With an observed mass of roughly $125 \mathrm{GeV}$, most important expected decay branching ratios and Higgs boson production cross-sections can be computed to the values listed in tables 1 and 2 .

SM Higgs boson branching ratios

\begin{tabular}{l|c|c|c|}
\hline Decay channel & BR (\%) & Decay channel & BR (\%) \\
\hline \hline$H \longrightarrow b \bar{b}$ & 58.24 & $H \longrightarrow W W$ & 21.4 \\
\hline$H \longrightarrow g g$ & 8.19 & $H \longrightarrow \tau \bar{\tau}$ & 6.27 \\
\hline$H \longrightarrow c \bar{c}$ & 2.89 & $H \longrightarrow Z Z$ & 2.6 \\
\hline$H \longrightarrow \gamma \gamma$ & 0.23 & $H \longrightarrow \mu \bar{\mu}$ & 0.02 \\
\hline
\end{tabular}

Table 1: Branching ratios (BR) at $m_{H}=125 \mathrm{GeV}$

\begin{tabular}{|c|c|c|c|c|c|}
\hline \multicolumn{6}{|c|}{ SM Higgs boson production cross-sections $\left(\mathrm{pb}^{-1}\right)$} \\
\hline$\sqrt{s}$ & ggF & VBF & WH & $\mathrm{ZH}$ & $\mathrm{ttH}$ \\
\hline $7 \mathrm{TeV}$ & 16.85 & 1.24 & 0.58 & 0.34 & 0.09 \\
\hline $8 \mathrm{TeV}$ & 21.40 & 1.60 & 0.70 & 0.42 & 0.13 \\
\hline $13 \mathrm{TeV}$ & 48.58 & 3.78 & 1.37 & 0.88 & 0.51 \\
\hline
\end{tabular}

Table 2: Production cross-sections at $m_{H}=125 \mathrm{GeV}$

In order to check if such predictions hold in ATLAS data, a global maximum likelihood fit is performed combining all the experimentally visible decay channels of the Higgs boson. In order to increase the sensitivity, it can be interesting to categorize events into different categories exploiting expected differences between Higgs boson decay events and backgrounds. Such categorization also provides a sensitivity to the Higgs production mechanisms. For instance, vector boson fusion events are expected to have jets in the forward/backward direction while vector boson associated production can have an extra lepton in the final state from $Z$ or $W$ decay. Minimally, a category holds all the events of a given final state so that minimum categorization corresponds to one category per final state. In each category, a number of signal events $n_{s}^{c}$ events is observed. On a general basis, this number can be related to the SM predicted Higgs boson production mechanism $i$ cross-section, $\sigma_{i_{S M}}$, and to the branching ratio of the related final state $f, B R_{f_{S M}}$, by the following formula:

$$
n_{s}^{c}=\sum_{i} \sum_{f} \mu_{i} \times \sigma_{i_{S M}} \times \mu_{f} \times B R_{f_{S M}} \times A_{i, f}^{c} \times \varepsilon_{i, f}^{c} \times \mathscr{L}^{c}
$$

where $\mathscr{L}^{c}$ is the luminosity of the set of events with the final state $f, A_{i, f}^{c}$ is the acceptance in that category for the events produced by the production mechanism $i$ and decaying in the final state $f, \varepsilon_{i, f}^{c}$ is the trigger and reconstruction efficiency of those events while $\mu_{i}$ and $\mu_{f}$ are the signal strength parameters that measure the agreement between observed data and SM predictions $\sigma_{i_{S M}}$ and $B R_{f_{S M}}$. If SM is predicting the observation, all $\mu$ parameters will be equal to one. Signal acceptances are computed using Monte Carlo samples and efficiencies are usually measured using data-driven techniques. Luminosity, acceptances and efficiencies suffer from systematic uncertainties evaluated by implementing in the fit nuisance parameters constrained by penalty log normal or gaussian functions using auxiliary measurements or Monte Carlo estimates as parameters. In each category, the number of observed events is a sum of signal and background events where the background event number is fitted using control regions dominated by background. The fit uses parametrized functions of final state variables to build the likelihood function. In the case of the diphoton final state for instance, it uses the invariant mass distribution of the diphoton events 
where the signal shape parameters are estimated from Monte Carlo samples and profiled in the likelihood maximization while background parameters are fitted directly from control regions, i.e. the sidebands around the Higgs mass peak. A similar strategy is used for the four-lepton final state case but depending on the category, the fit uses either multivariate analysis output distributions (jet categories) or just event counting (VH category) in the fit.

\section{Properties of the Standard Model Higgs boson with ATLAS LHC Run 1 data}

The ATLAS experiment in its configuration used during LHC Run 1 is described in details in [3]. ATLAS Run 1 dataset corresponds to an integrated luminosity of $25 \mathrm{fb}^{-1}$ taken at 7 and 8 $\mathrm{TeV}$ center-of-mass energies. Based on the analysis of the two more mass sensitive Higgs boson decay channels (diphoton and four-lepton final states), ATLAS measured the new boson mass to be $M_{H}=125.36 \pm 0.37$ (stat.) \pm 0.18 (syst.) GeV[4]. A combined fit of ATLAS and CMS LHC Run 1 data allowed to measure this mass to be $M_{H}=125.09 \pm 0.21$ (stat.) \pm 0.11 (syst.) GeV[5]. LHC Run 1 data allowed to test coupling and production cross-section SM predictions using a global fit of the various observed final states and allowed to provide self consistency of the SM within a precision of 20 to $40 \%$ depending on the observable decay channel [6]. The same data allowed also to directly observe the new boson in single decay channels demonstrating its coupling to SM model vector bosons ( $\gamma \gamma[7], Z Z[8]$ and $W W[9])$ and to SM fermions ( $\tau \tau[10]$ and indirectly to the top quark since the observed new boson production rate is seen with a cross-section as predicted by SM which is dominated by a top quark loop). Finally, this dataset has allowed to show that the new boson was unlikely to differ from a $J^{P}=0^{+}$state as predicted by the SM[11]. Run 1 dataset also provided a first set of Higgs production differential cross-sections[12].

After LHC Run 1, the expected SM Higgs sector has been checked to an interesting precision but few predictions remain to be verified: mainly its coupling to $b$ quark, to muon and electron, its decay to $Z \gamma$ and the evidence of its auto-coupling through a double Higgs boson production processes. The $t t H$ and $V H$ production process are also still to be measured with a significant accuracy.

\section{Properties of the Standard Model Higgs boson with ATLAS LHC Run 2 data}

For Run 2 period, the ATLAS detector has been upgraded to face the increasing luminosity of the LHC with the addition of an extra layer of silicon detector at very low radius to allow a better b-tagging and a better collision vertex reconstruction. The gas mixture used inside the transition radiation tracker (TRT) has been changed to significantly minimize losses. New chambers were also added in the muon spectrometer and the calorimeter readout has been consolidated. The forward detectors were modified to improve the measurement of the LHC luminosity, and a new aluminum beam pipe was installed to reduce the background. The whole ATLAS readout system was improved to be able to run at $100 \mathrm{kHz}$. Simultaneously, data acquisition software and monitoring applications were re-engineered. In particular, the trigger system was redesigned, going from 3 levels to 2 while implementing smarter and faster selection algorithms. ATLAS event reconstruction time also was also reduced in spite of the larger activity in the detector. A very ambitious 
upgrade of simulation, reconstruction and analysis software was completed, and a new generation of data management tools on the GRID was implemented.

Up to mid-july 2016, ATLAS accumulated a dataset of $13.3 \mathrm{fb}^{-1}$ of diphoton events and $14.8 \mathrm{fb}^{-1}$ of four-lepton events. This dataset has been used to perform a first check of SM predictions at $13 \mathrm{TeV}$. This analysis is performed in four different steps. In a first step, a direct global test of the SM is done using the inclusive diphoton and four-lepton samples by fitting a single global production cross-section $\sigma_{p p \rightarrow H+X}$ assuming SM branching ratios and SM acceptances. The measurement of the total Higgs cross-section $\sigma_{p p \rightarrow H+X}=59.0_{-9.2}^{+9.7}(\text { stat. })_{-3.5}^{+4.4}($ syst. $) \mathrm{pb}$ is found to be compatible with SM prediction $\sigma_{p p \rightarrow H+X}^{S M}=55.5_{-3.4}^{2.4} \mathrm{pb}$. Fig.1 shows the corresponding measured Higgs boson total production cross-section for $13 \mathrm{TeV}$ as measured in each channel and the value from the combined fit together with the previous measurements done at 7 and $8 \mathrm{TeV}$.

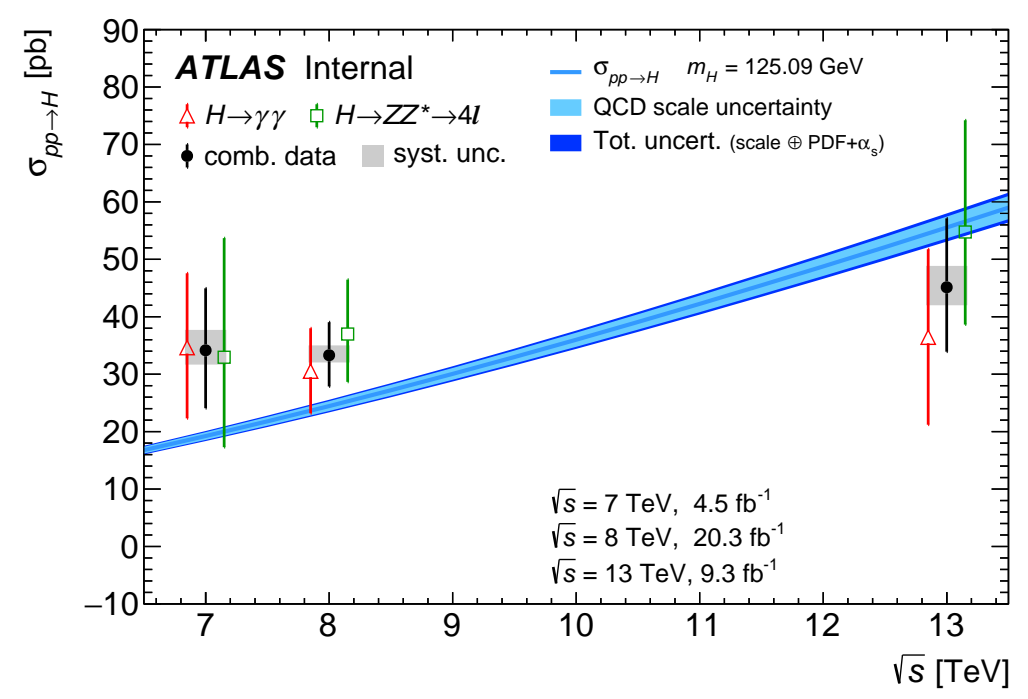

Figure 1: Total $p p \rightarrow H+X$ cross sections measured at different centre-of-mass energies compared to Standard Model predictions at up to $\mathrm{N}^{3} \mathrm{LO}$ in QCD. The red triangles show the measurements from the $H \rightarrow \gamma \gamma$ channel, the green rectangles show the measurements from the $H \rightarrow Z Z^{*} \rightarrow 4 \ell$ channel, and the black dots show the combinations of these two channels. The grey bands on the combined measurements represent the systematic uncertainty, while the black lines are the total uncertainty. The SM predictions (for a Higgs boson mass of $125.09 \mathrm{GeV}$ ) are shown as a smooth curve [13].

The same dataset categorized in five and thirteen categories for diphoton and four-lepton final states respectively allows to get a better sensitivity to Higgs boson production mechanisms. Several fits allowing a different number of degrees of freedom are then performed along the strategy defined in table 3. The first fit of the categorized events assumes a common value of $\mu_{i, f}=\mu$ and results into a measurement compatible with SM prediction $\mu=1$ :

$$
\mu=1.13_{-0.17}^{+0.18}
$$

In a second fit, Eq. 1.1 can be rewritten as a function of three fitted parameters $(\sigma . B)_{g g F}^{Z Z}$, the gluon-gluon fusion (ggF) Higgs boson production cross-section times the $H \rightarrow Z Z^{*}$ branching ratio, $\sigma_{V B F} / \sigma g g F$, the ratio of vector boson fusion $(\mathrm{VBF})$ and $g g F$ production cross-sections and 
Table 3: Summary of the fit strategies based on event categorisation

\begin{tabular}{ll}
\hline \hline & $\begin{array}{l}\text { Number of } \\
\text { parameters of interest }\end{array}$ \\
\hline Global signal strength $\mu$ & 1 \\
$(\sigma \cdot \mathrm{B})_{g \mathrm{ggF}}^{Z Z}, \sigma_{\mathrm{VBF}} / \sigma_{\mathrm{ggF}}$ and $\mathrm{B}{ }^{\gamma \gamma} / \mathrm{B}^{Z Z}$ & 3 \\
Independent $\sigma_{\dot{i}}$ assuming SM B & 5 \\
Independent $\sigma_{i} \cdot \mathrm{B}^{f}$ & 7 \\
\hline \hline
\end{tabular}

$B^{\gamma \gamma} / B^{Z Z}$, the ratio between branching ratios of Higgs decays to diphoton and two $\mathrm{Z}$ bosons. In a third fit, Eq. 1.1 can be rewritten as a function of five fiducial production cross-sections $\left(\left|y_{H}\right|<2.5\right)$ assuming SM branching ratios while in a fourth fit, one assumes seven independent cross-section $\times$ branching ratio terms. The plots corresponding to these three different fit results are presented in Fig. 2a, Fig. 2b and Fig. 2c and show no significative deviation from SM predictions.

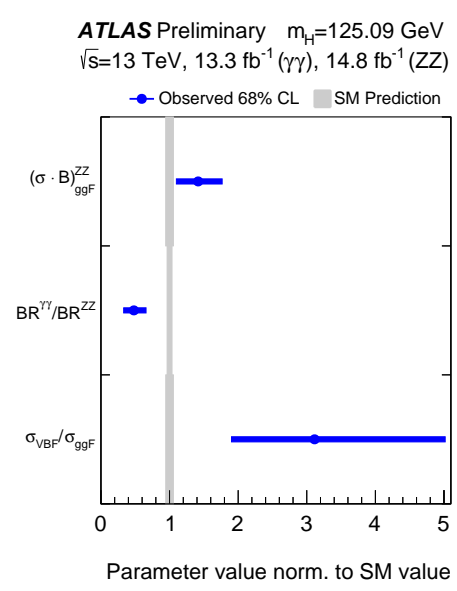

(a) Fit result with 3 parameters

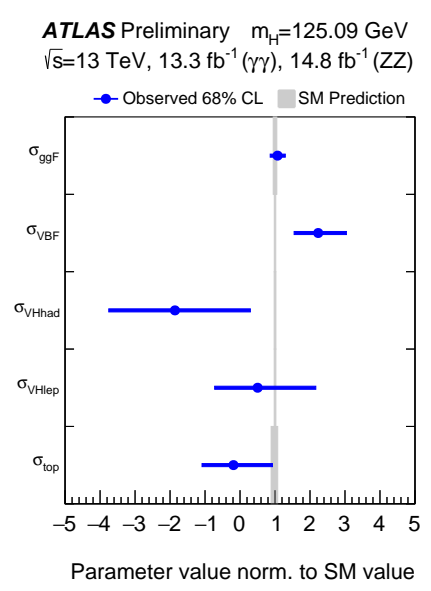

(b) Fit result with 5 parameters

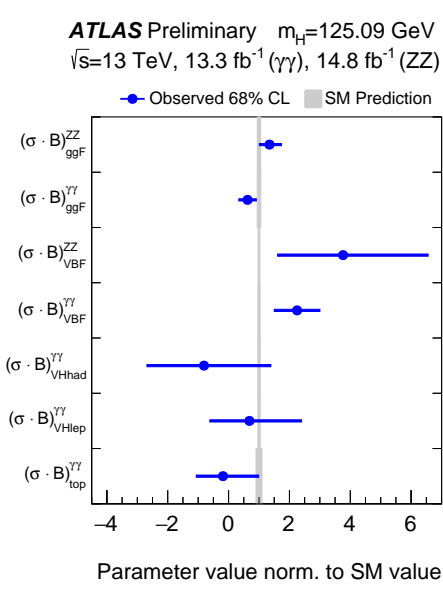

(c) Fit result with 7 parameters

Figure 2: Plots showing the results of the last three different fits presented in table 3 in units of the SM predictions.[13]

\section{Conclusion}

Combined measurements based on Higgs boson production cross sections and branching ratios using proton-proton collision data produced by the LHC at $\sqrt{s}=13 \mathrm{TeV}$ and recorded by the ATLAS detector have been presented based on the measurements performed in the individual $H \rightarrow \gamma \gamma$ and $H \rightarrow Z Z^{*}$ decay channels. Higgs boson production is observed in the $13 \mathrm{TeV}$ dataset with a local significance of about $10 \sigma$ (8.6 $\sigma$ expected $)$, and evidence for production via vector boson fusion is observed with a local significance of about $4 \sigma(1.9 \sigma$ expected). Extra fits allowing possible deviations from the SM predictions show no significant deviation. 
LHC Run 2 data will grow quickly until end of 2016 to approximatively 30 to $40 \mathrm{fb}^{-1}$ and should allow to test further the Higgs sector with a higher precision than LHC Run 1.

\section{References}

[1] ATLAS Collaboration, "Observation of a new particle in the search for the Standard Model Higgs boson with the ATLAS detector at the LHC ", Physics Letters B, Volume 716 (2012) 1

[2] CMS collaboration, "Observation of a new boson at a mass of $125 \mathrm{GeV}$ with the $\mathrm{CMS}$ experiment at the LHC", Physics Letters B, Volume 716 (2012) 30

[3] ATLAS Collaboration, "The ATLAS Experiment at the CERN Large Hadron Collider", 2008 JINST 3 S08003.

[4] ATLAS collaboration, "Measurement of the Higgs boson mass from the $H \rightarrow \gamma \gamma$ and $H \rightarrow Z Z^{*} \rightarrow 4 \ell$ channels in pp collisions at center-of-mass energies of 7 and $8 \mathrm{TeV}$ with the ATLAS detector", Phys. Rev. D 90, 052004, 2014

[5] ATLAS and CMS collaborations, "Combined Measurement of the Higgs Boson Mass in pp Collisions at $\sqrt{s}=7$ and $8 \mathrm{TeV}$ with the ATLAS and CMS Experiments", Physics Review Letters 114, 19

[6] ATLAS collaboration, "Measurements of the Higgs boson production and decay rates and coupling strengths using pp collision data at $\sqrt{s}=7$ and $8 \mathrm{TeV}$ in the ATLAS experiment", Eur. Phys. J. C (2016) 76: 6.

[7] ATLAS collaboration, "Measurement of Higgs boson production in the diphoton decay channel in $p p$ collisions at center-of-mass energies of 7 and $8 \mathrm{TeV}$ with the ATLAS detector", Phys. Rev. D. 90, 112015 (2014)

[8] ATLAS collaboration, "Search for the Standard Model Higgs boson in the decay channel $H \rightarrow Z Z^{*} \rightarrow 4 \ell$ with $4.8 \mathrm{fb}^{-1}$ of pp collisions at $\sqrt{s}=7 \mathrm{TeV}$ with ATLAS", Phys.Lett. B710 (2012) $383-402$

[9] ATLAS collaboration, "Study of (W/Z)H production and Higgs boson couplings using H ? WW* decays with the ATLAS detector", J. High Energ. Phys. (2015) 2015: 137.

[10] ATLAS collaboration, "Evidence for the Higgs-boson Yukawa coupling to tau leptons with the ATLAS detector", JHEP 04 (2015) 117

[11] ATLAS collaboration, "Study of the spin and parity of the Higgs boson in diboson decays with the ATLAS detector", Eur. Phys. J. C75 (2015) 476

[12] The ATLAS collaboration, "Measurements of the Total and Differential Higgs Boson Production Cross Sections Combining the $H \rightarrow \gamma \gamma$ and $H \rightarrow Z Z^{*} \rightarrow 4 \ell$ Decay Channels at $\sqrt{s}=8 \mathrm{TeV}$ with the ATLAS Detector ", Phys. Rev. Lett. 115 (2015) 091801

[13] ATLAS Collaboration, "Combined measurements of the Higgs boson production and decay rates in $H \rightarrow Z Z^{*} \rightarrow 4 \ell$ and $H \rightarrow \gamma \gamma$ final states using pp collision data at $\sqrt{s}=13 \mathrm{TeV}$ in the ATLAS experiment", ATLAS-CONF-2016-081, http://cds.cern.ch/record/2206272 